\title{
Efeito de fungicidas no controle de Fusarium graminearum, germinação, emergência e altura de plântulas em sementes de trigo.
}

\author{
Daniel Garcia Júnior ${ }^{1}$; Marta Helena Vechiato ${ }^{2}$; José Otávio Machado Menten ${ }^{3}$
}

\begin{abstract}
${ }^{1,3}$ Departamento de Entomologia, Fitopatologia e Zoologia Agrícola, USP/ESALQ, caixa postal, 13418-900, Piracicaba, SP; ${ }^{2}$ CPDSV - Instituto Biológico de São Paulo, caixa postal 12898-12959, 04010-970. São Paulo, SP; * Parte da tese de doutorado do primeiro autor.

Autor para correspondência: Daniel Garcia Júnior

Data de chegada: 16/10/2006. Aceito para publicação em: 29/03/2008
\end{abstract}

1408

\section{RESUMO}

Garcia Júnior, D., Vechiato, M. H., Menten, J. O.M. Efeito de fungicidas no controle de Fusarium graminearum, germinação, emergência e altura de plântulas em sementes de trigo. Summa Phytopathologica, v.34, n.3, p.280-283, 2008

Visando avaliar o efeito do tratamento químico na incidência de Fusarium graminearum, bem como na germinação, emergência e altura de plântulas, sementes de trigo da cultivar BR 18 Terena foram tratadas com os seguintes fungicidas nas respectivas doses de i.a./ 100 $\mathrm{kg}$ de sementes: captana $(150,0 \mathrm{~g})$, tiofanato metílico $(75,0 \mathrm{~mL})$, triflumizole $(45,0 \mathrm{~g})$, triticonazole $(45,0 \mathrm{~mL})$, triadimenol $(13,5 \mathrm{~mL})$, tolyfluanida $(75,0 \mathrm{~g})$, tebuconazole $(5,0 \mathrm{~mL})$, fludioxonil $(5,0 \mathrm{~mL})$, difeconazole $(30,0 \mathrm{~mL})$ e thiabendazole $(30,0 \mathrm{~mL})$. Em laboratório os fungicidas triflumizol, triadimenol, triticonazole, thiabendazole e tiofanato metílico reduziram significativamente a incidência de $F$. graminearum nas sementes em relação à testemunha. Thiabendazole e tiofanato metílico foram superiores ao triflumizol e triadimenol; os demais fungicidas comportaram-se de maneira semelhantes à testemunha. Não houve influência dos fungicidas na germinação das sementes. Em casa de vegetação a emergência e velocidade de emergência de plântulas, também não foram afetadas. Por outro lado, triadimenol interferiu negativamente na altura de plântulas aos sete DAS e captana, trticonazole, tebuconazole e triadimenol aos 14 DAS.

Palavras-chave adicionais: Giberela; fungicidas.

\section{ABSTRACT}

Garcia Júnior, D., Vechiato, M. H., Menten, J. O.M. Effects of fungicides on Fusarium graminearum control, germination, emergency and e height of seedlings in wheat seeds. Summa Phytopathologica, v.34, n.3, p.280-283, 2008

The effect of chemical treatment of wheat seeds (cv. "BR 18 Terena") was studied under laboratory and greenhouse conditions using the following fungicides with the respective doses of i.a./100 kg of seeds: captan $(150 \mathrm{~g})$, methilic tioafanate $(75 \mathrm{~mL})$, triflumizole $(45$ $\mathrm{g})$, triticonazole $(45 \mathrm{~mL})$ triadimenol $(13.5 \mathrm{~mL})$, tolyfluanid $(75 \mathrm{~g})$, tebuconazole $(5 \mathrm{~mL})$, fludioxonil $(5 \mathrm{~mL})$, difeconazole $(30 \mathrm{~mL})$ and thiabendazole $(30 \mathrm{~mL})$. In laboratory, seeds health and germination were evaluated while under greenhouse conditions were evaluated emergency, velocity of emergency and the height of seedlings. The fungicides triflumizole, triadimenol, triticonazole, thiabendazole and methilic tioafanate reduced significantly the incidence of $F$. graminearum in the seeds with thiabendazole and methilic tioafanate being the most efficient ones. Seeds germination, emergence and velocity of the seedlings were not affected by chemical treatment with the fungicides. On the other hand, triadimenol affect negativity the height of seedlings to 7 days after sowing and captan, triticonazole, tebuconazole and triadimenol to 14 days.

Additional keywords: Fusarium head bligth; fungicides.

No Brasil, a giberela cujo agente causal é o fungo Gibberella zeae (Schw.) Petch (anam. Fusarium graminearum Schwabe), a qual está associada às sementes, alcançou o "status" de principal doença nas regiões tritícolas, principalmente na região Sul do país (4)

Apesar desta doença estar amplamente disseminada nas regiões produtoras de trigo, o plantio constante de sementes contaminadas com F.graminearum pode aumentar a fonte de inoculo no campo, podendo em condições favoráveis ao patógeno causar grandes prejuízos. Portanto a utilização de sementes livres do patógeno é uma medida que visa diminuir a fonte de inoculo.

De acordo com Machado (7), o melhor método para o controle de doenças é sempre o uso de sementes de boa qualidade. Quando não há disponibilidade dessas sementes em quantidade suficiente, ou se deseja introduzir materiais de procedências duvidosas, ou ainda reduzir o potencial de inóculo primário, é recomendável que as sementes sejam tratadas. No caso de fungos de solo, o tratamento das sementes pode também proteger as plântulas (12).

O tratamento químico é o método mais comum de se tratar sementes, sendo de grande valor comercial. O seu princípio é bastante simples e baseia-se na existência de produtos eficientes contra o(s) patógeno(s), que apresentem baixa fitotoxicidade e sejam pouco tóxicos ao homem e ao ambiente (8).

O presente trabalho teve como objetivo avaliar o efeito do tratamento químico de sementes de trigo (Triticum aestivum L.) na incidência de Fusarium graminearum, bem como verificar possíveis efeitos, positivos e negativos, na germinação, emergência e altura de 
plântulas de trigo.

Sementes da cultivar BR 18 Terena cedidas pelo Centro Nacional de Pesquisa de Trigo (CNPT - EMBRAPA Trigo/Passo Fundo - RS), apresentando $25 \%$ de incidência de $F$. graminearum, foram tratadas com os seguintes fungicidas nas respectivas doses de i.a./ $100 \mathrm{~kg}$ de sementes: captana $(150,0 \mathrm{~g})$, tiofanato metílico $(75,0 \mathrm{~mL})$, triflumizole $(45,0 \mathrm{~g})$, triticonazole $(45,0 \mathrm{~mL})$, triadimenol $(13,5 \mathrm{~mL})$, tolyfluanida $(75,0 \mathrm{~g})$, tebuconazole $(5,0 \mathrm{~mL})$, fludioxonil $(5,0 \mathrm{~mL})$, difeconazole $(30,0 \mathrm{~mL})$ e thiabendazole $(30,0 \mathrm{~mL})$. Após os tratamentos das sementes, realizou-se dois experimentos, um de laboratório e um de casa-de-vegetação. Em laboratório foram avaliadas a germinação e a sanidade das sementes. Em casa-de-vegetação avaliou-se o efeito dos tratamentos fungicidas na altura de plântulas e emergência. Em seguida, determinou-se o índice de velocidade de emergência (IVE), de acordo com a fórmula descrita por Maguire (14): IVE $=\mathrm{G} 1 / \mathrm{N} 1+\mathrm{G} 2 / \mathrm{N} 2+\ldots$ $+\mathrm{Gn} / \mathrm{Nn}$, onde $\mathrm{G} 1, \mathrm{G} 2, \mathrm{Gn}=$ número de plântulas normais na primeira, segunda e enésima ("n") contagem; e N1, N2, Nn = número de dias após a semeadura na primeira, segunda e enésima ("n") contagem.

A incidência de Fusarium graminearum e de outros fungos nas sementes utilizadas foi avaliada utilizando o método do papel de filtro modificado com congelamento $(5,3)$, empregando delineamento inteiramente casualizado, com 11 tratamentos e quatro repetições de 50 sementes. Após o período de incubação, as sementes foram examinadas, com o auxílio de microscópio estereoscópio determinandose a incidência dos fungos presentes nas sementes.

A germinação das sementes foi avaliada empregando-se o método do rolo de papel (3). O delineamento estatístico utilizado foi o inteiramente casualizado com quatro repetições de 50 sementes cada. A germinação foi determinada contando-se o número de plântulas normais, oito dias após a incubação, sendo o resultado expresso em porcentagem.

No experimento em casa de vegetação foi empregado o delineamento inteiramente casualizado, com 11 tratamentos e quatro repetições de 50 sementes. Cada unidade experimental constitui-se de uma caixa de plástico medindo $45 \times 30 \times 11 \mathrm{~cm}$, contendo areia tratada com brometo de metila. Foram avaliadas as seguintes variáveis: (i) velocidade de emergência - contagem de plântulas aos dois, três, cinco e dez dias após a emergência (D.A.E); (ii) altura de plântulas - mensuração do comprimento da parte aérea de dez plântulas, escolhidas ao acaso, em cada tratamento, realizada aos sete, 14 e 21 dias após a emergência; e (iii) avaliação de plântulas sintomáticas - as plântulas foram retiradas da areia, colocadas em câmara úmida por cinco dias e posteriormente analisadas com o auxílio de microscópio estereoscópio para verificação de sinais do patógenos. Quando necessário, procedeu-se com o isolamento em meio de cultura BDA (batata-dextrose-ágar).

Os dados obtidos, tanto nos experimentos de laboratório quanto os de casa-de-vegetação, foram submetidos à análise de variância e submetidos ao teste de Tukey, com o auxílio "software" STAT ${ }^{\circledR}$.

Os resultados dos testes de laboratório apresentados na Tabela 1 mostram que o triflumizol, triadimenol, triticonazole, thiabendazole e tiofanato metílico controlaram $F$. graminearum, reduzindo significativamente a sua incidência nas sementes em relação à testemunha. Os fungicidas thiabendazole e tiofanato metílico foram superiores ao triflumizol e triadimenol. Tebuconazole, captana, difenoconazole, tolyfluanida e fludioxonil comportaram-se de maneira semelhantes à testemunha. Resultados semelhantes no controle desse patógeno foram observados por Moraes et al. (9) ao utilizarem triadimenol, o qual também apresentou eficiência no controle da giberela e fludioxonil que se mostrou ineficiente no mesmo controle.

Resultados interessantes foram encontrados para os fungicidas tiofanato metílico, triticonazole, triadimenol e triflumizol. Embora estes fungicidas não sejam recomendados para o controle de $F$. graminearum em sementes de trigo, os mesmos apresentaram eficiência de $100 \%, 76 \%, 62 \%$ e $61 \%$, respectivamente, em relação à incidência do patógeno na testemunha (Tabela 1). Tais resultados indicam a eficiência dessas moléculas na inibição ou mesmo ação fungistática das moléculas sobre o desenvolvimento do fungo. Barreto et al. (2) estudaram o efeito de fungicidas sobre Fusarium oxysporum f. sp. gladioli e verificaram que o fungicida tiofanato metílico promoveu a inibição do crescimento micelial deste patógeno em meio de cultura. Tal resultado reforça a eficiência dessa molécula no controle de $F$. graminearum.

Os resultados obtidos nos tratamentos com thiabendazole e tiofanato metílico, os quais indicam o controle da fusariose nas sementes, são corroborados por aqueles resultados obtidos por Teles Neto (13) que também verificou o controle do patógeno em sementes de trigo.

Os resultados apresentados na Tabela 1 mostram que não houve

Tabela 1. Porcentagem de fungos e de germinação em sementes de trigo, cultivar BR 18 Terena, tratadas ou não com fungicidas.

\begin{tabular}{|c|c|c|c|c|c|}
\hline Tratamento & Fusarium graminearum & Fusarium sp. & Bipolaris sp. & Bipolaris sorokiniana & Germinação \\
\hline Testemunha & $44,5 \mathrm{~A}$ & $2,0 \mathrm{~A}$ & $0,5 \mathrm{~A}$ & $1,0 \mathrm{~A}$ & $85,0 \mathrm{~A}$ \\
\hline Tebuconazole & $32,0 \mathrm{AB}$ & $0,5 \mathrm{~A}$ & $0,0 \mathrm{~A}$ & $0,5 \mathrm{~A}$ & $80,0 \mathrm{~A}$ \\
\hline Captana & $30,5 \mathrm{AB}$ & $3,0 \mathrm{~A}$ & $0,0 \mathrm{~A}$ & $0,0 \mathrm{~A}$ & $75,5 \mathrm{~A}$ \\
\hline Difeconazole & $29,0 \mathrm{AB}$ & $3,0 \mathrm{~A}$ & $0,5 \mathrm{~A}$ & $0,5 \mathrm{~A}$ & $85,5 \mathrm{~A}$ \\
\hline Tolyfluanida & $20,5 \mathrm{AB}$ & $2,0 \mathrm{~A}$ & $0,5 \mathrm{~A}$ & $0,0 \mathrm{~A}$ & $83,5 \mathrm{~A}$ \\
\hline Fludioxonil & $18,0 \mathrm{AB}$ & $1,5 \mathrm{~A}$ & $1,5 \mathrm{~A}$ & $0,0 \mathrm{~A}$ & $84,0 \mathrm{~A}$ \\
\hline Triflumizol & $17,5 \quad \mathrm{~B}$ & $0,5 \mathrm{~A}$ & $0,0 \mathrm{~A}$ & $0,0 \mathrm{~A}$ & $76,5 \mathrm{~A}$ \\
\hline Triadimenol & $17,0 \quad \mathrm{~B}$ & $1,5 \mathrm{~A}$ & $0,0 \mathrm{~A}$ & $0,0 \mathrm{~A}$ & $75,0 \mathrm{~A}$ \\
\hline Triticonazole & $10,5 \quad \mathrm{BC}$ & $0,0 \mathrm{~A}$ & $0,5 \mathrm{~A}$ & $0,0 \mathrm{~A}$ & $79,5 \mathrm{~A}$ \\
\hline Thiabendazole & 1,5 & $0,0 \mathrm{~A}$ & $1,0 \mathrm{~A}$ & $0,0 \mathrm{~A}$ & $81,5 \mathrm{~A}$ \\
\hline Tiofanato metílico & $0,0 \quad \mathrm{C}$ & $0,0 \mathrm{~A}$ & $0,0 \mathrm{~A}$ & $0,0 \mathrm{~A}$ & $84,5 \mathrm{~A}$ \\
\hline $\mathrm{F}$ & $11,2 * *$ & 1,5 n.s. & 1,1 n.s. & 1,5 n.s. & 1,4 n.s. \\
\hline C.V. $(\%)$ & 25,1 & 40,5 & 27,4 & 18,9 & 8,2 \\
\hline
\end{tabular}

C.V: coeficiente de variação; **: significativo pelo teste de F com $99 \%$ de confiança; n.s.: não significativo; valores acompanhados de letras diferentes (colunas) diferem estatisticamente entre si pelo teste de Tukey com 95\% de confiança. 
Tabela 2. Altura de plântulas, emergência de plântulas e índice de velocidade de emergência (IVE) provenientes de sementes de trigo, cultivar BR 18 Terena, tratadas ou não com diferentes fungicidas

\begin{tabular}{|c|c|c|c|c|c|}
\hline \multirow[t]{2}{*}{ Tratamento } & \multicolumn{3}{|c|}{ Altura de plântulas $(\mathrm{cm})$} & \multirow{2}{*}{$\begin{array}{c}\text { Emergência }(\%) \\
21 \text { DAS }\end{array}$} & \multirow[t]{2}{*}{ Índice de velocidade de emergência (IVE) } \\
\hline & 7 DAS & 14 DAS & 21 DAS & & \\
\hline Testemunha & $10,4 \mathrm{~A}$ & $25,2 \mathrm{~A}$ & $30,0 \mathrm{AB}$ & $39,0 \mathrm{AB}$ & $50,1 \mathrm{~A}$ \\
\hline Tolyfluanida & $10,5 \mathrm{AB}$ & $22,7 \mathrm{ABCD}$ & $27,9 \mathrm{AB}$ & $39,8 \mathrm{AB}$ & $50,8 \mathrm{~A}$ \\
\hline Difeconazole & $10,1 \mathrm{AB}$ & $23,6 \mathrm{AB}$ & $30,7 \mathrm{~A}$ & $37,4 \mathrm{AB}$ & $47,1 \mathrm{~A}$ \\
\hline Fludioxonil & $10,0 \mathrm{AB}$ & $23,2 \mathrm{ABC}$ & $28,8 \mathrm{AB}$ & $38,1 \mathrm{AB}$ & $48,3 \mathrm{~A}$ \\
\hline Thiabendazole & $9,8 \quad \mathrm{AB}$ & $23,7 \mathrm{AB}$ & $28,8 \mathrm{AB}$ & $37,3 \mathrm{AB}$ & $45,1 \mathrm{~A}$ \\
\hline Captana & $9,8 \quad \mathrm{AB}$ & $21,3 \quad \mathrm{BCD}$ & $25,5 \mathrm{AB}$ & $36,5 \quad \mathrm{~B}$ & $48,5 \mathrm{~A}$ \\
\hline Triflumizol & $9,5 \quad \mathrm{AB}$ & $23,0 \mathrm{ABC}$ & $30,2 \mathrm{AB}$ & $37,2 \mathrm{AB}$ & $45,0 \mathrm{~A}$ \\
\hline Triticonazole & $8,3 \mathrm{AB}$ & CDE & $25,2 \mathrm{AB}$ & $39,6 \mathrm{AB}$ & $50,7 \mathrm{~A}$ \\
\hline Tebuconazole & $8,0 \quad \mathrm{AB}$ & 19,3 & $25,3 \mathrm{~B}$ & $39,2 \mathrm{AB}$ & $48,4 \mathrm{~A}$ \\
\hline Tiofanato metílico & $9,7 \quad \mathrm{AB}$ & $23,1 \mathrm{ABC}$ & $28,6 \mathrm{AB}$ & $42,1 \mathrm{~A}$ & $46,6 \mathrm{~A}$ \\
\hline Triadimenol & $6,1 \quad \mathrm{~B}$ & 17,1 & $26,0 \mathrm{AB}$ & $36,4 \quad$ B & $43,7 \mathrm{~A}$ \\
\hline $\mathrm{F}$ & $2,9 * *$ & $10,8 * *$ & $3,4 * *$ & $2,9 *$ & 0,4 n.s. \\
\hline$\overline{\text { C.V. }(\%)}$ & 10,3 & 3,5 & 4,2 & 5,2 & 14,3 \\
\hline
\end{tabular}

DAS: dias após semeadura; n.s.: não significativo; **: diferença significativa com $99 \%$ de confiança pelo teste de F; C.V.: coeficiente de variação; valores acompanhados por letras diferentes (colunas) apresentam diferença significativa com 95\% de confiança pelo teste de Tukey.

diferenças significativas entre os tratamentos com fungicidas e a testemunha, indicando que não houve influência de nenhum dos produtos testados na germinação das sementes. Resultado semelhante, para o caso específico do fungicida triticonazole, foi observado por Picinini e Fernandes (10).

Moraes et al. (9) também não observaram melhora no desempenho de sementes de trigo da cultivar EMBRAPA 40 com o uso de fungicidas como difeconazole, triadimenol e fludioxonil.

Na emergência, avaliada aos 21 dias após a semeadura (DAS), embora não tenha sido constatada diferença significativa entre os tratamentos e a testemunha, observa-se que sementes tratadas com tiofanato metílico apresentaram emergência superior aquelas sementes tratadas com captana e triadimenol (Tabela 2). Lasca et al. (6) verificaram que o triadimenol promoveu o aumento da emergência de plântulas de trigo em condições de campo, contudo sua eficiência não foi das melhores encontradas para o controle de Bipolaris sorokiniana.

$\mathrm{O}$ tratamento com triadimenol reduziu significativamente a altura de plântulas aos sete e 14 dias após a semeadura (DAS). Captana, triticonazole, tebuconazole apresentaram valores significativamente inferiores em relação à testemunha. Apesar de não ter havido diferenças em relação à testemunha na aos 21 DAS, sementes tratadas com tebuconazole, originaram plântulas com altura significativamente inferior àquelas tratadas com difeconazole. Os fungicidas triadimenol e tebuconazole em experimentos realizados por Moraes et al. (9) e Balardin e Facco (1), respectivamente, mostraram efeito negativo na altura de plântulas. Tais resultados indicam que estes produtos podem apresentar efeito fitotóxico à plântula de trigo.

A Tabela 2 ainda permite observar que para o índice de velocidade de emergência (IVE), não houve diferenças significativas entre os tratamentos, indicando a ausência de efeitos negativos ou positivos.

É indiscutível a validade de se controlar Fusarium graminearum através do tratamento químico das sementes. Além de reduzir a quantidade de inóculo que será inserido no campo de produção, o controle de doenças de parte aérea via tratamento de sementes é uma nova alternativa, caracterizada pela facilidade de uso e do menor risco de contaminação ambiental, a qual poderá abrir novas perspectivas no controle das doenças em cereais de inverno no Brasil (10).
Os resultados obtidos no presente trabalho permitem concluir que: (i) fungicidas como triflumizol, triadimenol, triticonazole, thiabendazole e tiofanato metílico reduziram a incidência de $F$. graminearum em sementes de trigo; e (ii) os fungicidas avaliados não influenciaram na germinação, emergência e velocidade de emergência de plântulas.

\section{REFERÊNCIAS BIBLIOGRÁFICAS}

1. Balardin, R. S.; Facco, M. J. Eficiência e fitotoxicidade de fungicidas no controle de Helminthosporium sativum em sementes de trigo (Triticum aestivum) cultivar BR 23. Fitopatologia Brasileira, Brasília, v. 28, supl., p. 276, 2003. (Resumo).

2. Barreto, S . S.; Resende, M. L. V.; Boas, C. V. Efeito de fungicidas sobre Fusarium oxysporum f. sp. gladioli. Fitopatologia Brasileira, Brasília, v. 28, supl., p. 305, 2003. (Resumo).

3. Brasil. Regras para análise de sementes. Brasília: Ministério da Agricultura e Reforma Agrária, 1992. 365p.

4. Del Ponte, E. M.; Fernandes, J. M. C.; Pierobom, C. R.; Bergstrom, G. C. Giberela do trigo - aspectos epidemiológicos e modelos de previsão. Fitopatologia Brasileira, Brasília, v. 29, n. 6, p. 587-606, 2004.

5. International Seed Testing Association: International rules for seed testing. Proceedings International Seed Testing Association, Zürich, v. 31, n.1, p. 1-52, 1966.

6. Lasca, C. C.; Barros, B. C.; Valarini, P. J.; Castro, J. L.; Chiba, S. Ação de fungicidas em tratamento de sementes de trigo (Triticum aestivum L.) no controle de Helminthosporium sativum Pammel, King \& Bakke. Biológico, São Paulo, v. 51, n. 9, p. 225$231,1985$.

7. Machado, J. C. Padrões de tolerância de patógenos associados a sementes. Revisão Anual de Patologia de Plantas, Passo Fundo, v. 2, p. 229-263, 1994.

8. Menten, J. O. M. Importância do tratamento de sementes. In: MENTEN, J. O. M. Patógenos em sementes: detecção, danos e controle químico. Piracicaba: ESALQ/FEALQ 1991 , p. 203-224.

9. Moraes, M. H. D.; Menten, J. O. M.; Almeida, R. R.; Souza, L. Efeito do tratamento químico na qualidade sanitária e fisiológica de sementes de trigo. Informativo Abrates, Brasília, v. 7, n. 1/2, p. 141, 1997. (Resumo).

10. Picinini, E. C.; Fernandes, J. M. C. Controle de doenças do trigo. In: Bacaltchuk, G. R.; Org, B. Tecnologia para produ- 
zir trigo no Rio Grande do Sul. Porto Alegre: Assembléia legislativa, Comissão de Agricultura, Pecuária e Cooperativismo; Passo Fundo: EMBRAPA Trigo, 2000. cap. 2, p.13-27. (Série Culturas, 2)

11. Picinini, E. C.; Fernandes, J. M. C. Efeito do tratamento de sementes com fungicidas no controle de doenças na parte aérea da cultura do trigo em 1999. In: Simpósio Brasileiro de Patologia de Sementes, 7., Sete Lagoas. Resumos...: Sete Lagoas: EMBRA-
PA Milho e Sorgo, 2002. p.39.

12. Ribeiro, A. S. Tratamento de sementes com fungicidas. Revisão Anual de Patologia de Plantas, Passo Fundo, v. 4, p. 382, 1996.

13. Teles Neto, F. X. B. Transmissão e controle de Fusarium graminearum em sementes e danos causados pela giberela em trigo. 2004. 113p. Dissertação (Mestrado em Fitopatologia) - Universidade de Passo Fundo, Passo Fundo. 